\title{
In-depth proteomic analysis of Plasmodium berghei sporozoites using trapped ion mobility spectrometry with parallel accumulation-serial fragmentation
}

\author{
Soumia Hamada ${ }^{1,3}$ | Cédric Pionneau ${ }^{1}$ | Christophe Parizot ${ }^{2}$ | Olivier Silvie ${ }^{3}$ | \\ Solenne Chardonnet ${ }^{1} \quad$ | Carine Marinach ${ }^{3}$ (c) \\ ${ }^{1}$ Sorbonne Université, INSERM, UMS PASS, Plateforme Post-génomique de la Pitié Salpêtrière (P3S), Paris, France \\ ${ }^{2}$ Sorbonne Université, INSERM, Centre d'Immunologie et des Maladies Infectieuses, CIMI-Paris, Assistance Publique Hôpitaux de Paris, Hôpital Pitié-Salpêtrière, \\ Paris, France \\ ${ }^{3}$ Sorbonne Université, INSERM, CNRS, Centre d'Immunologie et des Maladies Infectieuses, CIMI-Paris, Paris, France
}

\section{Correspondence}

Carine Marinach, Sorbonne Université, INSERM, CNRS, Centre d'Immunologie et des Maladies Infectieuses, CIMI-Paris, F-75013 Paris, France.

Email: carine.marinach_patrice@sorbonneuniversite.fr

\begin{abstract}
Sporozoites of the malaria parasite Plasmodium are transmitted by mosquitoes and infect the liver for an initial and obligatory round of replication, before exponential multiplication in the blood and onset of the disease. Sporozoites and liver stages provide attractive targets for malaria vaccines and prophylactic drugs. In this context, defining the parasite proteome is important to explore the parasite biology and to identify potential targets for antimalarial strategies. Previous studies have determined the total proteome of sporozoites from the two main human malaria parasites, P. falciparum and $P$. vivax, as well as $P$. yoelii, which infects rodents. Another murine malaria parasite, $P$. berghei, is widely used to investigate the parasite biology. However, a deep view of the proteome of $P$. berghei sporozoites is still missing. To fill this gap, we took advantage of the highly sensitive timsTOF PRO mass spectrometer, combined with three alternative methods for sporozoite purification, to identify the proteome of $P$. berghei sporozoites using low numbers of parasites. This study provides a reference proteome for $P$. berghei sporozoites, identifying a core set of proteins expressed across species, and illustrates how the unprecedented sensitivity of the timsTOF PRO system enables deep proteomic analysis from limited sample amounts.
\end{abstract}

KEYWORDS

malaria, mass spectrometry, Plasmodium berghei, proteome, sporozoites, timsTOF PRO

\footnotetext{
Abbreviations: AMA1, apical membrane antigen 1; AP2, apetala 2; CD81, cluster of differentiation 81; CelTOS, cell-traversal protein for ookinetes and sporozoites; CERLI1, cytosolically exposed rhoptry leaflet interacting protein 1; CLAMP, claudin-like apicomplexan microneme protein; CSP, circumsporozoite protein; DG, density gradient; GEST, gamete egress and sporozoite traversal protein; GFP, green fluorescent protein; MACS, magnetic-activated cell sorting; MAEBL, membrane associated erythrocyte binding-like protein; MTRAP, merozoite TRAP-like protein; PABA, para-aminobenzoic acid; PASEF, parallel accumulation serial fragmentation; PLP1, perforin-like protein 1; RASP2, rhoptry apical surface protein 2; RhopH, high molecular weight rhoptry protein; RON, rhoptry neck protein; SLARP, sporozoite and liver stage asparagine-rich protein; SPECT, sporozoite protein essential for cell traversal; SR-B1, scavenger receptor class B type 1; SUB, subtilisin-like protein; TIMS, trapped ion mobility spectrometry; TRAP, thrombospondin-related anonymous protein; TREP, TRAP-like protein

This is an open access article under the terms of the Creative Commons Attribution License, which permits use, distribution and reprodu the original work is properly cited.

(c) 2021 The Authors. Proteomics published by Wiley-VCH GmbH
} 


\section{1 | INTRODUCTION}

Malaria, with more than 200 million estimated cases and 400,000 deaths every year [1], remains a major public health problem in many countries. Significant progress has been achieved over the past decades in reducing malaria incidence and mortality, through the systematic use of insecticide-treated bednets and potent antimalarial artemisinin-based drug combinations. However, progress in malaria control has recently stalled, and the continued emergence of parasite and mosquito resistance to antimalarial medicines and insecticides, respectively, is a serious threat to malaria control. The disease is caused by parasites of the genus Plasmodium, which are transmitted via the bite of infected Anopheles female mosquitoes. Invasive forms called sporozoites present in the salivary glands of the vector are deposited in the dermis during a blood meal. After traversing the skin, motile sporozoites traffic to the liver through the blood stream, and reach the liver parenchyma where they invade hepatocytes for an initial and obligatory replication phase, resulting in the release of tens of thousands of merozoites [2]. These merozoites invade erythrocytes and initiate the exponential asexual reproduction of the parasite in erythrocytes, causing the symptomatic phase of malaria. Of the Plasmodium species infecting humans, Plasmodium falciparum is the most prevalent and the deadliest species, especially in subsaharan Africa. P. vivax, the second most important species in humans, is widely distributed around the world but causes less severe malaria. One particularity of $P$. vivax is to cause relapsing malaria episodes, due to hypnozoites, which are dormant liver-stage parasites that can reactivate weeks or months after parasite transmission by a mosquito.

Infection of the liver by sporozoites is an essential and clinically silent phase of the malaria life cycle, and has long been considered as an ideal target for a malaria vaccine [3]. However, despite intense research efforts, no efficacious malaria vaccine has been licensed yet. Until now, a single antigen, the circumsporozoite protein (CSP), has been pursued as a vaccine target against the extracellular sporozoite stage. The principal malaria vaccine candidate, RTS,S, targets CSP from P. falciparum, yet confers only partial and short-lived protection [4]. This emphasizes the need to develop more efficacious malaria vaccines targeting other antigens. In this context, proteomic studies of sporozoites, by identifying the set of proteins expressed by these forms, can guide the downselection of potential new candidates.

Working with limited sample amounts has long been a challenge for in-depth proteome analysis. Studying the Plasmodium sporozoite proteome magnifies such a problem. Indeed, sporozoites can only be obtained from infected mosquitoes and must be isolated by hand dissection of the insect salivary glands. In addition, sporozoites are small cells (around $1 \mu \mathrm{m} \times 10-15 \mu \mathrm{m}$ ) that can be obtained only in limited numbers (usually less than $10^{5}$ per mosquito). Recovery of high numbers of sporozoites for downstream proteomic studies typically requires dissecting hundreds of mosquitoes, resulting in a massive contamination of the samples with mosquito material. Methods have been developed to purify sporozoites and limit the proportion of mosquito proteins [5]. These approaches, combined with LC-MS/MS, led to the characterization of the total proteome of $P$. falciparum and $P$. vivax sali-

\section{Statement of significance}

Plasmodium sporozoites are the mosquito-transmitted forms of the malaria parasite. Here we report a proteomic analysis of sporozoites of Plasmodium berghei, a widely used rodent malaria model parasite. By employing a highly sensitive mass spectrometry approach based on trapped ion mobility spectrometry with parallel accumulation-serial fragmentation, we identified the deep proteome of $P$. berghei sporozoites using unprecedented low numbers of parasites, and identified a core set of sporozoite proteins expressed across Plasmodium species. Our study illustrates how the high sensitivity of the timsTOF PRO system enables deep proteomic analysis from limited sample amounts.

vary gland sporozoites, where the most comprehensive studies identified 2039 and 1972 proteins, respectively (out of more than 5500 proteins encoded by their genome) [6-10]. Similar studies performed in the rodent $P$. yoelii identified 1774 proteins in salivary gland sporozoites of this species $[8,10]$. Surface proteomes were also described for the three species, based on chemical labeling of live parasites followed by LC-MS/MS [8, 9, 11].

$P$. berghei is another rodent malaria parasite that has been widely used as a model to study Plasmodium pre-erythrocytic stages. P. berghei is closely related to $P$. yoelii yet shows differences in the invasion routes used by sporozoites to infect hepatocytes. While $P$. yoelii, like $P$. falciparum, relies on the host protein CD81 to infect hepatocytes [12], $P$. berghei can use the scavenger receptor B1 (SR-B1) as an alternative entry route $[13,14]$. As SR-B1 is also involved during $P$. vivax infection [13], P. berghei provides a suitable model to study SR-B1-dependent liver infection [15]. The molecular basis of this differential usage of host receptors is not fully elucidated, but the sporozoite 6-cysteine domain protein P36 was shown to play a central role [13]. Although P. berghei is the most studied rodent parasite, the sporozoite proteome for this species has been solved only partially, with 134 proteins identified in one report [16]. Importantly, in the current version of PlasmoDB (v49) [17], proteins with reported mass spectrometry evidence in $P$. berghei sporozoites correspond to an extrapolation from proteomic studies performed with $P$. yoelii sporozoites [8].

Here, we took advantage of the highly sensitive timsTOF PRO mass spectrometer to determine the total proteome of $P$. berghei sporozoites. This system is equipped with the trapped ion mobility spectrometry (TIMS) technology for parallel accumulation serial fragmentation (PASEF). The dual TIMS technology enables ions trapping in the front section and ions separation according to their ion mobility in the rear section. Combined with rapid quadrupole switching and TOF detector, the timsTOF PRO enables the fragmentation of multiple simultaneously eluting precursor ions with a near $100 \%$ duty cycle. The timsTOF PRO offers speed and sensitivity gains of up to 10 -fold compared to 
PbGFP-infected Anopheles stephensi mosquitoes

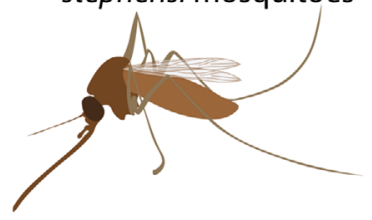

Hand dissection of salivary gland
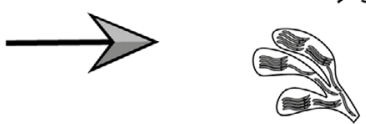

PbGFP sporozoites, mosquito material, microbiota

\section{Sporozoite Purification}

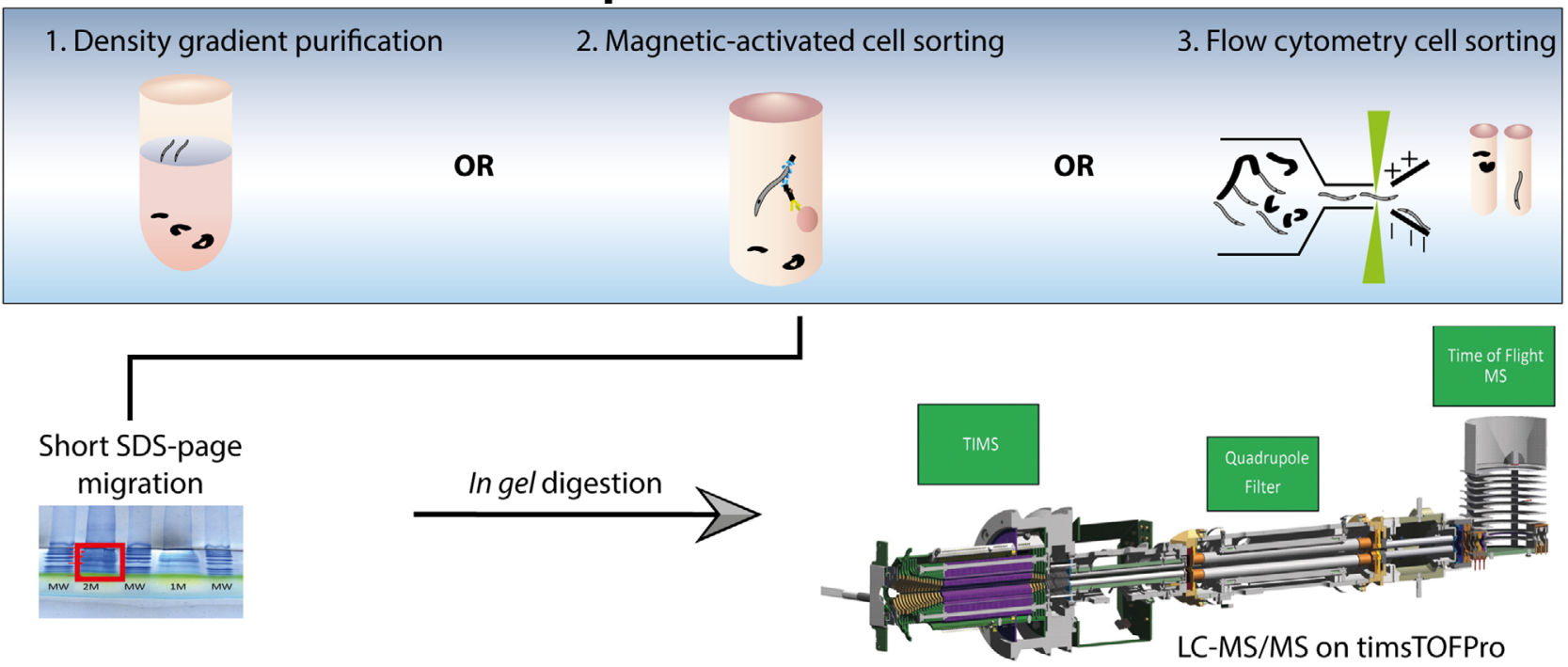

FIG URE 1 Workflow for sample preparation and proteomic analysis. GFP-expressing P. berghei sporozoites were collected by hand dissection of the salivary glands of infected A. stephensi mosquitoes, and purified either by density gradient centrifugation, immunocapture with magnetic beads or by flow cytometry cell sorting. Lysates from purified sporozoites were processed by SDS-PAGE and in-gel trypsin digestion, and resulting peptide samples were analyzed by LC-MS/MS with the TimsTOF Pro system

other mass spectrometry approaches [18]. Combined with three alternative methods for sporozoite purification, this system allowed us to identify the deep proteome of $P$. berghei sporozoites using unprecedented low numbers of parasites.

\section{RESULTS AND DISCUSSION}

\subsection{Efficiency of sporozoite purification}

Plasmodium sporozoites are obtained by hand dissection of the salivary glands of infected mosquitoes, resulting in sample contamination with proteins from the mosquito or its microbiota. Purification of sporozoites after dissection is a crucial step to reduce the quantity of contaminating proteins of mosquito origin. We compared three different methods for parasite purification: 1) the density gradient purification procedure, developped by Kennedy et al. [5], used as a reference method 2) immunocapture of sporozoites using magnetic beads coupled to anti-CSP antibodies and 3) sorting of fluorescent sporozoites by flow cytometry. Figure 1 illustrates the workflow that was used for the proteomic analysis of $P$. berghei sporozoites. In total, four independent sporozoite preparations were purified with the density gradient method, two using magnetic beads and four by flow cytometry, as sum- marized in Table 1. For each purification method, we assessed both the sporozoite recovery rate, that is, the proportion of sporozoites recovered after purification, and the efficiency of the purification at the protein level. For this purpose, lysates of purified sporozoites were analyzed by LC-MS/MS to determine the proportion of parasite- versus mosquito-derived proteins.

Purification using the density gradient protocol was relatively easy to execute but the sporozoite recovery rate was highly variable, ranging from 9-39\% (mean 28\%) (Table 1). Despite this lack of reproducibility in our hands, the purification efficiency was satisfying, with P. berghei proteins representing between 30 and 55\% (mean 39.93\%) of the total number of proteins identified by mass spectrometry (Figure 2A), which is consistent with values reported with other species [8]. Immunocapture of sporozoites with anti-CSP antibodies coupled to magnetic beads was easy and rapid to execute. However, mosquito debris remained abundant despite extensive bead washes. In addition, as sporozoites were agglutinated with the beads after elution, it was impossible to determine the yield of recovered sporozoites with this method. Nevertheless, samples were analyzed by mass spectrometry, resulting in around 35\% of identified proteins being of parasite origin (Table 1 and Figure 2A). Sorting of GFP-expressing sporozoites by flow cytometry also showed highly variable recovery rates, varying between 10 and 55\% (Table 1). We hypothesize that the poor 
TAB LE 1 Summary of the samples analyzed in this study

\begin{tabular}{|c|c|c|c|c|c|c|c|c|}
\hline Experiments & $\begin{array}{l}\text { Initial } \\
\text { sporozoite } \\
\text { number }\end{array}$ & $\begin{array}{l}\text { Purification } \\
\text { method }\end{array}$ & $\begin{array}{l}\text { Sporozoite } \\
\text { recovery } \\
\text { rate }^{a}\end{array}$ & $\begin{array}{l}\text { Number of } \\
\text { sporozoites } \\
\text { on gel }^{\text {b }}\end{array}$ & Run & $\begin{array}{l}\text { Number of } \\
\text { sporozoites } \\
\text { injected }^{c}\end{array}$ & $\begin{array}{l}\text { Number } \\
\text { of } \\
\text { replicates }\end{array}$ & $\begin{array}{l}\% \text { P. berghei } \\
\text { proteins }^{d}\end{array}$ \\
\hline \multirow[t]{3}{*}{ DG1 } & $9.3 \mathrm{M}$ & DG & $24 \%$ & $100 \mathrm{k}$ & DG1a & $25 k$ & 2 & $\begin{array}{l}26.3 \% \\
26.7 \%\end{array}$ \\
\hline & & & & $1.0 \mathrm{M}$ & DG1b & $100 k$ & 3 & $\begin{array}{l}31.4 \% \\
31.9 \% \\
32 \%\end{array}$ \\
\hline & & & & & DG1c & $200 k$ & 3 & $\begin{array}{l}32.9 \% \\
31.8 \% \\
29.4 \%\end{array}$ \\
\hline DG2 & $2.3 \mathrm{M}$ & DG & $39 \%$ & $900 \mathrm{k}$ & DG2 & $900 \mathrm{k}$ & 1 & $41.8 \%$ \\
\hline DG3 & $2.3 \mathrm{M}$ & DG & $9 \%$ & $200 k$ & DG3 & $200 k$ & 1 & $32.2 \%$ \\
\hline DG4 & $2.8 \mathrm{M}$ & DG & $39 \%$ & $1.09 \mathrm{M}$ & DG4 & $1.09 \mathrm{M}$ & 1 & $55.4 \%$ \\
\hline MA1 & 18.8M & MA & $N^{e}$ & $2.0 \mathrm{M}^{\mathrm{f}}$ & MA1 & $2.0 \mathrm{M}^{\mathrm{f}}$ & 1 & $35 \%$ \\
\hline MA2 & $1.8 \mathrm{M}$ & MA & $\mathrm{ND}^{\mathrm{e}}$ & $1.8 \mathrm{M}^{\mathrm{e}}$ & MA2 & $900 k^{f}$ & 1 & $34 \%$ \\
\hline FC1 & $2.0 \mathrm{M}$ & $\mathrm{FC}$ & $15 \%$ & $290 k$ & FC1 & $145 k$ & 2 & $\begin{array}{l}67 \% \\
69 \%\end{array}$ \\
\hline $\mathrm{FC} 2$ & 8.0M & $\mathrm{FC}$ & $8 \%$ & $320 k$ & $\mathrm{FC} 2$ & $320 k$ & 1 & $58 \%$ \\
\hline FC3 & $7.3 \mathrm{M}$ & $\mathrm{FC}$ & $10 \%$ & $350 k$ & FC3 & $350 k$ & 1 & $56 \%$ \\
\hline FC4 & $9.0 \mathrm{M}$ & FC & $55 \%$ & $5.0 \mathrm{M}$ & $\mathrm{FC} 4$ & $5.0 \mathrm{M}$ & 1 & $46.6 \%$ \\
\hline
\end{tabular}

DG, density gradient centrifugation; FC: flow cytometry cell sorting; MA, magnetic-activated cell sorting.

${ }^{\text {aP }}$ Proportion of sporozoites recovered after purification.

bEquivalent number of sporozoites in the sample loaded on the SDS-PAGE.

${ }^{\mathrm{c} E q u i v a l e n t}$ number of sporozoites analyzed in each LC-MS/MS run.

${ }^{\mathrm{d}}$ Proportion of $P$. berghei proteins among all proteins identified in each LC-MS/MS run.

eND, not determined.

${ }^{\mathrm{f} B e c a u s e}$ the number of sporozoites recovered with the magnetic-activated cell sorting procedure could not be determined, the indicated sporozoite numbers correspond to the maximal number of parasites analyzed assuming a $100 \%$ recovery rate.

recovery rate observed with some of the samples is due to the intrinsic low efficiency of the sorting method and to the fact that sorted parasites were highly diluted during the procedure, increasing the risk of parasite loss during subsequent centrifugations. Nevertheless, the purification efficiency was satisfying, with $P$. berghei proteins representing up to $60 \%$ of the total number of proteins identified by mass spectrometry (Table 1 and Figure 2A). Based on these results, we conclude that both immunocapture and FACS sorting of sporozoites provide valuable alternative to the density gradient reference method.

\subsection{Effect of sporozoite sample size on proteome identification}

We next analyzed the effect of sample size on the performance of the timsTOF PRO system for protein identification, for the three purification methods. Remarkably, mass spectrometry analysis of as few as $100,000-200,000$ sporozoites purified by the density gradient method (DG1 and DG3 samples in Table 1) identified 500-900 P. berghei proteins (Figure 2B), and a single analysis of 900,000 sporozoites (DG2 sample in Table 1) resulted in the identification of 1532 P. berghei pro- teins (Figure 2B), which represents $93 \%$ of the total number of proteins identified in the entire study (see below).

When combining all the identification results from 10 independent experiments (18 injections in Table 1), we detected a total of 1648 proteins in P. berghei salivary gland sporozoites (Data S1 and Data S2). Increasing the number of technical replicates (multiple injections of the same sample) had a modest impact on the number of proteins identified (Figure 2C), with an overlap above $80 \%$, which is more than previously reported on Orbitrap systems (59 to 76\%) [19, 20], and might be explained by the high frequency of the selection/fragmentation cycle (up to 100 per cycle). In contrast, increasing the number of biological independent samples increased substantially the number of proteins identified in $P$. berghei sporozoites (Figure 2D), with an overlap of only $30.7 \%$. The total number of proteins identified in $P$. berghei sporozoites is within the same range as those reported in previous proteomic studies of $P$. falciparum, $P$. vivax, and $P$. yoelii sporozoites (Figure 3A). However, 0.4-1 $\times 10^{7}$ purified salivary gland sporozoites were used for MS experiments in those studies [8-10], that is, 10 times more than reported here. Altogether, these results illustrate that the high sensitivity of the timsTOF PRO mass spectrometer allows in-depth identification of the Plasmodium sporozoite proteome, using low numbers of parasites. 
A

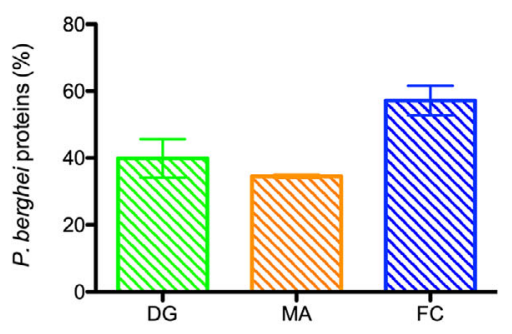

B

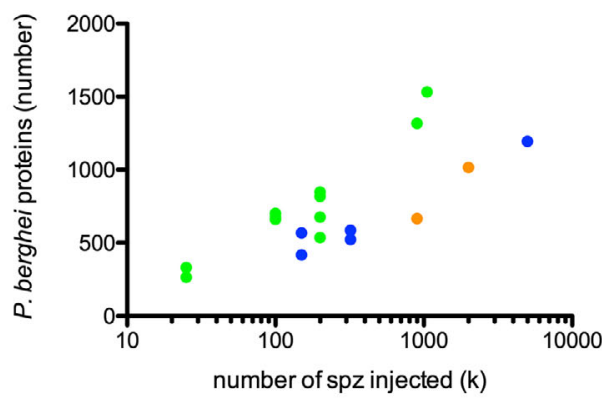

$D G$ : Gradient density purification

MA : Magnetic-activated cell sorting

FC : Flow cytometry cell sorting

C

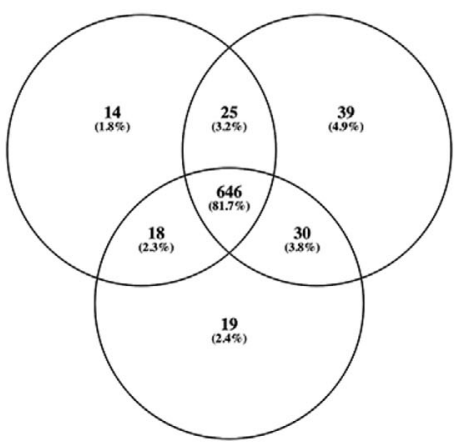

D

DG2 (1318) DG4 (1532)

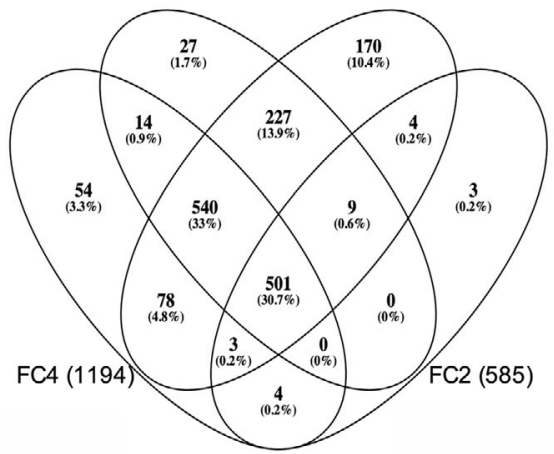

FIG URE 2 Effect of parasite purification and sample size on proteome identification. (A) The efficiency of sporozoite purification was evaluated at the protein level by determining the proportion of $P$. berghei proteins among all proteins identified by mass spectrometry. The data are displayed as the mean (+/-SEM) from two to four experiments for each purification method. (B) Number of $P$. berghei proteins identified by mass spectrometry depending on the sample size, represented as the equivalent number of sporozoites analyzed by mass spectrometry. Each data point corresponds to one MS/MS run. DG, density gradient centrifugation (green); FC, flow cytometry cell sorting (blue); MA, magnetic activated cell sorting (orange). (C) Venn diagram showing the number of $P$. berghei proteins identified in three technical replicates of the DG1a sample analysis (100,000 sporozoites). (D) Venn diagram showing the number of $P$. berghei proteins identified in four biological samples, each analyzed in a single run

\section{3 | Identification of a core set of sporozoite proteins across Plasmodium species}

Among the 1648 proteins identified in P. berghei sporozoites (Data S2), 1559 proteins (94.2\%) have been identified in at least one other species (Figure 3B and Data S3). More precisely, 1402 proteins (84.8\%) have been detected in P. falciparum, 1403 (84.9\%) in P. vivax and 1388 (83.9\%) in P. yoelii, showing overall extensive overlap. Comparison of the proteome datasets across species revealed a core set of 1204 proteins detected in salivary gland sporozoites from the four Plasmodium species (Data S3). In contrast, 51 (3.0\%) proteins were identified only in sporozoites from the rodent malaria species ( $P$. yoelii and $P$. berghei) and 89 (5.4\%) only in P. berghei.

\subsection{Analysis of sporozoite protein families}

We next scrutinized the $P$. berghei proteome dataset for a selection of protein families, in comparison with published proteomes from $P$. falciparum, P. vivax and P. yoelii (Data S3). Like other apicomplexan invasive stages, sporozoites possess specialized apical secretory organelles termed micronemes and rhoptries, whose regulated secretion plays a key role during parasite locomotion, migration through the host tissues and invasion of hepatocytes. Overall, micronemal and rhoptry proteins were well conserved between sporozoite species. Many wellcharacterized micronemal proteins were shared across the sporozoite proteome datasets, including TRAP family members (TRAP, TREP), proteins involved in cell traversal (SPECT, PLP1, CelTOS and GEST), 
A

\begin{tabular}{|c|c|c|c|}
\hline Species & $\begin{array}{l}\text { Number of } \\
\text { identified } \\
\text { proteins }\end{array}$ & $\begin{array}{l}\text { Sporozoite } \\
\text { numbers }\end{array}$ & References \\
\hline P. falciparum & 2039 & $\approx 10^{7}$ & [6] \\
\hline P. vivax & 1972 & $\approx 4 \times 10^{6}$ & [7] \\
\hline P. yoelii & 1774 & $\approx 10^{7}$ & [6] \\
\hline P. berghei & 1648 & $10^{5}-10^{6}$ & This study \\
\hline
\end{tabular}

B

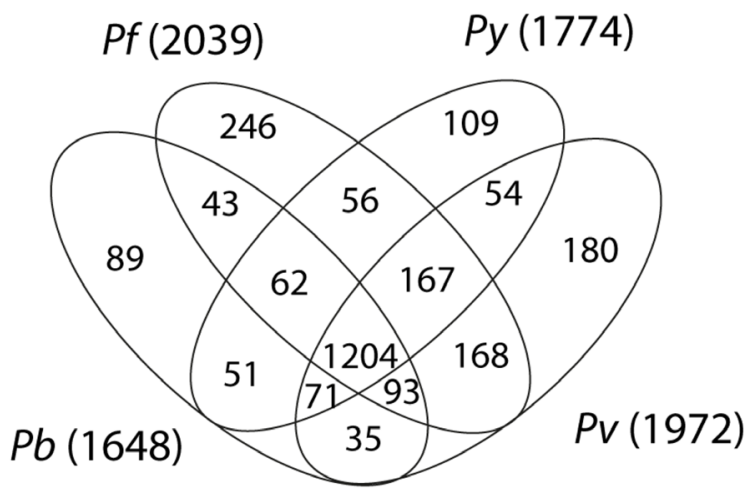

FIGURE 3 Comparison of the sporozoite proteomes across Plasmodium species. (A) Summary of proteomic studies of P. falciparum, $P$. vivax, $P$. yoelii, and $P$. berghei sporozoites, indicating the number of parasite proteins identified and the sample size. (B) Venn diagram representation of the sporozoite proteome composition across Plasmodium species

AMA1 and MAEBL, and members of the 6-cysteine domain (6-cys) protein family (P36, P52, P38 and B9). The claudin-like apicomplexan microneme protein (CLAMP), recently identified as a conserved apicomplexan protein that is essential for host cell invasion by Toxoplasma gondii tachyzoites and P. falciparum merozoites [21], was also identified in sporozoites from the four species, yet its role in this stage is still unknown. Eight rhoptry neck (RON) proteins were identified across sporozoite species (RON1/ASP, RON2, RON3, RON4, RON5, RON6, RON11 and RON12), as well as the rhoptry bulb high molecular weight rhoptry proteins (RhopH) 2 and 3.

We also noted some differences between sporozoite proteomes. For example, the merozoite TRAP-like protein (MTRAP) was identified in $P$. berghei, $P$. vivax, and $P$. yoelii but not in $P$. falciparum sporozoite samples. MTRAP is essential for gamete egress and formation of oocysts in the mosquito [22], but its role in sporozoites is unknown. The 6-cys protein $\mathrm{P} 12 \mathrm{p}$ was detected in $P$. berghei, $P$. yoelii and $P$. falciparum, but not in P. vivax. Among rhoptry proteins, the Cytosolically Exposed Rhoptry Leaflet Interacting protein 1 (CERLI1), was identified in P. berghei and P. vivax sporozoites only. CERLI1 (also referred to as RASP2) plays an essential role in P. falciparum merozoites and T. gondii tachyzoites for rhoptry secretion and host cell invasion [23, 24]. Subtilisin-like (SUB) proteins are essential serine proteases that cleave merozoite membrane proteins during invasion and egress [25, 26]. Interestingly, SUB2 was identified in sporozoites from $P$. falciparum, $P$. vivax and $P$. yoelii, but not $P$. berghei. Conversely, SUB1 was detected in sporozoites from $P$. berghei but not in the other species. When looking at factors involved in gene regulation, we observed variations in the repertoire of AP2 transcription factors detected in the different sporozoite populations. While seven AP2 factors were detected in at least three species (including the previously characterized AP2-SP/EXP, AP2-I, AP2-L, AP2-O4 and AP2-O5), 4 members of the family were exclusively detected in $P$. falciparum sporozoites (including AP2-O), and one in P. vivax (AP2-O2). The sporozoite and liver stage asparagine-rich protein (SLARP), a master regulator of liver stage development $[27,28]$, was identified in P. falciparum, P. berghei, and $P$. yoelii, but not in $P$. vivax. Whether these differences reflect biological specificities or merely variations in protein expression and/or detection remains to be determined. Finally, 137 proteins among the 1205 core set (11\%) correspond to proteins of unknown function.

\section{3 | CONCLUDING REMARKS}

This study provides a reference proteome dataset for $P$. berghei sporozoites, complementing previous proteomic studies of $P$. falciparum, $P$. vivax, and $P$. yoelii parasites. We identify a conserved set of more than 1200 sporozoite proteins shared between the four species, and found some differences between the datasets. Further investigations will be required to determine whether these differences reflect biological specificities or merely variations in protein abundance. Finally, identification of the $P$. berghei sporozoite proteome was achieved with much lower numbers of cells as compared to previous studies. This illustrates how the high sensitivity of the timsTOF PRO system enables deep proteomic analysis from limited sample amounts. In the context of malaria, this opens novel perspectives to explore the parasite proteome, including in elusive stages such as the hypnozoites, or for proteogenomic studies of field isolates.

\section{EXPERIMENTAL SECTION}

\subsection{Ethics statement}

All animal work was conducted in strict accordance with the Directive 2010/63/EU of the European Parliament and Council 'On the protection of animals used for scientific purposes'. Protocols were approved by the Ethical Committee Charles Darwin №005 (project \#7475).

\section{2 | Production of Plasmodium sporozoite-infected mosquitoes}

We used GFP-expressing P. berghei (PbGFP, ANKA strain) parasites, obtained after integration of a GFP expression cassette at the dispensable p230p locus [29]. PbGFP blood stage parasites were propa- 
gated in female Swiss mice (6-8 weeks old, from Janvier Labs). Anopheles stephensi mosquitoes were fed on PbGFP-infected mice using standard methods, and kept at $21^{\circ} \mathrm{C}$. Infected mosquitoes were fed on an $10 \% \mathrm{w} / \mathrm{v}$ sucrose solution water, supplemented with $0.05 \% \mathrm{w} / \mathrm{v}$ paraamino benzoic acid (PABA), and kept at $21^{\circ} \mathrm{C}$ under $70 \%$ humidity. Mosquitoes were collected and killed in $70 \%$ ethanol, and rinced in Leibovitz's L-15 Medium (supplemented with fungisone, penicillin and streptomicin). Salivary glands were individually isolated 21 days after the mosquito blood meal by microdissection, and collected in DMEM medium supplemented with antibiotics. Sporozoites were released by manual grinding of salivary glands, passed on a $40 \mu \mathrm{m}$ filter to eliminate mosquitoes large debris, and washed in DMEM. Collected sporozoites were kept at $4^{\circ} \mathrm{C}$.

\subsection{Density gradient purification of P. berghei sporozoites}

Density gradient purification was performed as described [5]. Briefly, a $17 \% \mathrm{w} / \mathrm{v}$ solution of Accudenz (Accurate Chemical \#AN7050) dissolved in distilled deionized water $\left(\mathrm{ddH}_{2} \mathrm{O}\right)$ was filter sterilized and stored at $4^{\circ} \mathrm{C}$. A $3 \mathrm{~mL}$ Accudenz cushion was loaded in a $15 \mathrm{~mL}$ conical tube and the dissected sporozoite mixture (up to $1 \mathrm{~mL}$ ) was gently layered on top of the cushion. The column was spun at $2500 \mathrm{~g}$ at $15^{\circ} \mathrm{C}$ for $20 \mathrm{~min}$ (no brake) and the interface was transferred to a new, clean microcentrifuge tube and spun at top speed in a microcentrifuge for $3 \mathrm{~min}$. The supernatant was discarded and the pelleted sporozoites were resuspended in PBS and counted in a Neubauer chamber.

\subsection{Immunocapture of P. berghei sporozoites}

Freshly dissected PbGFP sporozoites were incubated for $1 \mathrm{~h}$ at $4^{\circ} \mathrm{C}$ in DMEM in the presence of $10 \mu \mathrm{g} / \mathrm{mL}$ of the anti-CSP mAb 3D11 [30]. After centrifugation, pelleted sporozoites were incubated with Protein $\mathrm{G}$ coupled MACS microbeads for $15 \mathrm{~min}$ at $4^{\circ} \mathrm{C}$ according to the manufacturer's protocol. Beads were then trapped on MACS MS column and washed five times with PBS. Beads and sporozoites were then eluted, centrifuged and resuspended in PBS.

\subsection{Flow cytometry sorting of PbGFP sporozoites}

Sorting of PbGFP sporozoites was performed on a S3 cell sorter (Bio-Rad), using a $488 \mathrm{~nm}$ excitation, while fluorescence emission was detected using band pass filters of 530/30. Area and height of the forward scatter signal were used to exclude doublets, and recovery of PbGFP sporozoites was performed using a purity sortmask. The purity of the sporozoite samples was systematically verified by microscopy after sorting. Of note, flow cytometry sorting was performed on unfixed parasites in order to allow optimal protein extraction. Sorted sporozoites were collected in $1 \mathrm{X}$-PBS at $4^{\circ} \mathrm{C}$, centrifuged $\left(8000 \times \mathrm{g}, 10 \mathrm{~min}, 4^{\circ} \mathrm{C}\right)$, and stored at $-80^{\circ} \mathrm{C}$ until protein extraction.

\subsection{Parasite lysis and sample preparation for mass spectrometry}

Lysis of sporozoites was done directly in Laemmli buffer $(62.5 \mathrm{mM}$ Tris/HCl pH 6.8, 2\% w/v SDS, 10\% v/v glycero, 0.01\% w/v Bromophenol blue and $25 \mathrm{mM} \mathrm{DTT}$ ). Lysates were incubated at $90^{\circ} \mathrm{C}$ for $10 \mathrm{~min}$, centrifuged $\left(15,000 \times \mathrm{g}, 15 \mathrm{~min}, 4^{\circ} \mathrm{C}\right)$, and stored at $-80^{\circ} \mathrm{C}$ before analysis. Lysates were loaded on a polyacrylamide gel (SDS-PAGE) composed of a concentration gel ( $4 \%$ acrylamide) and a separation gel ( $10 \%$ acrylamide). A short electrophoresis migration was made (about $5 \mathrm{~mm}$ ). The gel was then fixed and stained with Coomassie blue Imperial Protein Stain (Thermo Fischer) for $2 \mathrm{~h}$. The short lanes containing the samples were then cut into small $1 \mathrm{~mm}^{3}$ pieces and placed on a 96-well pierced plate. Digestion was done by the DigestProMSi robot (Intavis). The samples were destained with a solution of $50 \mathrm{mM}$ ammonium bicarbonate $(\mathrm{AmBic})$ and $50 \%$ ethanol $(\mathrm{EtOH})$ at $60^{\circ} \mathrm{C}$. This step was followed by reduction (incubation in $10 \mathrm{mM}$ DTT in $50 \mathrm{mM} \mathrm{AmBic}$ for $30 \mathrm{~min}$ at $56^{\circ} \mathrm{C}$ ), and alkylation (incubation in $50 \mathrm{mM}$ iodoacetamide in $50 \mathrm{mM} \mathrm{AmBic}$ for $30 \mathrm{~min}$ at RT in the dark). Proteins were digested with $200 \mathrm{ng}$ trypsin per well overnight at $37^{\circ} \mathrm{C}$. The gel pieces were washed twice in $60 \%$ acetonitrile (ACN)/0.1\% trifluoroacetic acid (TFA) for 20 min. Peptide extracts were then dried with Speed-Vac and resuspended in $20 \mu \mathrm{L}$ of ACN 2\%/formic acid (FA) $0.1 \%$. Sample desalting was performed with home-made StageTips consisting in a stack of two reverse-phase C18 layers (Empore SPE Disks C18, Sigma Aldrich) inserted in a $10 \mu \mathrm{L}$ tip. This step was carried out using the DigestProMSi robot (Intavis) and was necessary before LC-MS/MS analysis to avoid fouling of the analytical column due to residual debris. The StageTip was first hydrated with methanol and then activated by passing a 50\% ACN/0.5\% acetic acid (HAc) solution and then a 0.5\% HAc solution. The peptide solution previously diluted with $0.5 \%$ acetic acid to a final volume of $70 \mu \mathrm{L}$ was then gently passed through the StageTip. The peptides retained in the StageTip were washed and desalted by passing the $0.5 \% \mathrm{HAc}$ solution. Finally, the peptides were eluted with an $\mathrm{ACN} 80 \% / \mathrm{HAc} 0.5 \%$ solution, completely dried (Speed-Vac) and resolubilized in $20 \mu \mathrm{L}$ of ACN 2\%/FA 0.1\%. Samples were stored at $-20^{\circ} \mathrm{C}$ until MS analysis. If necessary, peptide samples were concentrated using Speed-Vac to reduce the volume before injection into the LC-MS/MS. In some cases, we also used the pre-column (reverse phase) integrated into the LC-MS/MS system for on-line peptide desalting.

\subsection{Liquid chromatography tandem mass spectrometry}

Peptides were analyzed by the new and sensitive timsTOF PRO mass spectrometer (Bruker) coupled to the nanoElute HPLC. Peptides were 
separated on an Odyssey column from lonOpticks (1.6 $\mu \mathrm{m}$ C18, $120 \AA$, $75 \mu \mathrm{m} \mathrm{ID}, 25 \mathrm{~cm}$ ) using a 90 min gradient from 2 to $37 \%$ ACN with $0.1 \%$ formic acid. MS acquisition was run in DDA mode with PASEF, from 100 to $1700 \mathrm{~m} / \mathrm{z}$ with an active exclusion of $0.4 \mathrm{~min}$. Parent ion selection was achieved with a two-dimensional $\mathrm{m} / \mathrm{z}$ and $1 / \mathrm{k} 0$ selection area filter allowing exclusion of singly charged ions. Low-abundance precursors were selected several times for PASEF-MS/MS until the target value. Capillary voltage was set at $1,4 \mathrm{kV}$. The total cycle time was $1.15 \mathrm{~s}$ with 10 PASEF cycles.

\subsection{Data collection and analysis}

To compare our data with data from a previous proteomic analysis of $P$. yoelii sporozoites $[8,10]$, all data were processed in the same way. Mascot generic files (mgf) were generated using Data Analysis 5.1 (Bruker) and processed with X!Tandem pipeline version 0.2.36 (http://pappso.inra.fr/bioinfo/xtandempipeline) using databases from UniProt or PlasmoDB. The databases used were: Contaminants_20160129 containing a list of common contaminants, Anopheles_stephensi_UP.fasta (Date 09/2018, number of entries: 11,767) and PlasmoDB_PB_39_PbergheiANKA (Date 09/2018, number entered: 5076). The search parameters were: maximum one missed cleavage allowed, Cys-CAM as fixed modification, Met-Ox and acetyl $\mathrm{N}$-term as variable modifications. MS mass error tolerances were adapted to instrument. MS1 mass error tolerance were set to $50 \mathrm{ppm}$ for timsTOF PRO and 25 ppm for Orbitrap. MS2 mass error tolerance were set to $50 \mathrm{ppm}$ for timsTOF PRO and 0,4 Da for Orbitrap. Protein lists were filtered and validated using Proline software version 1.6 .1 ( http: //www.profiproteomics.fr/proline ) with FDR $<1 \%$ at peptide and protein level and with a minimum of one peptide per protein.

\section{ACKNOWLEDGMENTS}

The authors thank Jean-François Franetich, Maurel Tefit and Thierry Houpert for rearing of mosquitoes and technical assistance. This work was supported in part by the Laboratoire d'Excellence ParaFrap (ANR-11-LABX-0024), the Agence Nationale de la Recherche (ANR16-CE15-0004 and ANR-16-CE15-0010) and the Fondation pour la Recherche Médicale (EQU201903007823). The authors acknowledge the Conseil Régional d'Ile-de-France, Sorbonne Université, the National Institute for Health and Medical Research (INSERM) and the Biology, Health and Agronomy Infrastructure (IBiSA) for funding the timsTOF PRO.

\section{CONFLICT OF INTEREST}

The authors declare no conflict of interest.

\section{DATA AVAILABILITY STATEMENT}

The mass spectrometry proteomics data have been deposited to the ProteomeXchange Consortium (http://proteomecentral.proteome xchange.org) via the PRIDE partner repository [31] with the dataset identifier PXD022735.
Data S1, Supporting Information lists of all proteins (1a) and peptides (1b) identified in extracts from infected mosquito salivary glands after parasite purification.

Data S2, Supporting Information lists the P. berghei proteins identified in purified sporozoite samples.

Data S3, Supporting Information compares the proteomes of $P$. falciparum, $P$. vivax, $P$. yoelii, and $P$. berghei sporozoites.

\section{ORCID}

Carine Marinach (D) https://orcid.org/0000-0002-8667-2208

\section{REFERENCES}

1. World Health Organization (2019). World Malaria Report 2019, 1232.

2. Frischknecht, F., \& Matuschewski, K. (2017). Plasmodium sporozoite biology. Cold Spring Harbor Perspectives in Medicine, 7, a025478.

3. Duffy, P. E., \& Patrick Gorres, J. (2020). Malaria vaccines since 2000: progress, priorities, products. NPJ Vaccines, 5, 1-9.

4. RTS, S Clinical Trials Partnership (2015). Efficacy and safety of RTS,S/AS01 malaria vaccine with or without a booster dose in infants and children in Africa: Final results of a phase 3, individually randomised, controlled trial. Lancet, 386, 31-45.

5. Kennedy, M., Fishbaugher, M. E., Vaughan, A. M., Patrapuvich, R., Boonhok, R., Yimamnuaychok, N., Rezakhani, N., Metzger, P., Ponpuak, M., Sattabongkot, J., Kappe, S. H., Hume, J. C., \& Lindner, S. E. (2012). A rapid and scalable density gradient purification method for Plasmodium sporozoites. Malaria Journal, 11:421, 1-10.

6. Florens, L., Washburn, M. P., Raine, J. D., Anthony, R. M., Grainger, M., Haynes, J. D., Moch, J. K., Muster, N., Sacci, J. B., Tabb, D. L., Witney, A. A., Wolters, D., Wu, Y., Gardner, M. J., Holder, A. A., Sinden, R. E., Yates, J. R., \& Carucci, D. J. (2002). A proteomic view of the Plasmodium falciparum life cycle. Nature, 419, 520-526.

7. Lasonder, E., Janse, C. J., Van Gemert, G. J., Mair, G. R., Vermunt, A M. W., Douradinha, B. G., Van Noort, V., Huynen, M. A., Luty, A. J. F., Kroeze, H., Khan, S. M., Sauerwein, R. W., Waters, A. P., Mann, M., \& Stunnenberg, H. G. (2008). Proteomic profiling of Plasmodium sporozoite maturation identifies new proteins essential for parasite development and infectivity. PLoS Pathogens, 4, e1000195.

8. Lindner, S. E., Swearingen, K. E., Harupa, A., Vaughan, A. M., Sinnis, P., Moritz, R. L., \& Kappe, S. H. I. (2013). Total and putative surface proteomics of malaria parasite salivary gland sporozoites. Molecular and Cellular Proteomics, 12, 1127-1143.

9. Swearingen, K. E., Lindner, S. E., Flannery, E. L., Vaughan, A. M., Morrison, R. D., Patrapuvich, R., Koepfli, C., Muller, I., Jex, A., Moritz, R. L., Kappe, S. H. I., Sattabongkot, J., \& Mikolajczak, S. A. (2017). Proteogenomic analysis of the total and surface-exposed proteomes of Plasmodium vivax salivary gland sporozoites. PLoS Neglected Tropical Diseases, 11, e0005791.

10. Lindner, S. E., Swearingen, K. E., Shears, M. J., Walker, M. P., Vrana, E. N., Hart, K. J., Minns, A. M., Sinnis, P., Moritz, R. L., \& Kappe, S. H. I. (2019). Transcriptomics and proteomics reveal two waves of translational repression during the maturation of malaria parasite sporozoites. Nature Communications, 10, 4964.

11. Swearingen, K. E., Lindner, S. E., Shi, L., Shears, M. J., Harupa, A., Hopp, C. S., Vaughan, A. M., Springer, T. A., Moritz, R. L., Kappe, S. H. I., \& Sinnis, P. (2016). Interrogating the Plasmodium sporozoite surface: Identification of surface-exposed proteins and demonstration of glycosylation on CSP and TRAP by mass spectrometry-based proteomics. PLoS Pathogens, 12, e1005606.

12. Silvie, O., Rubinstein, E., Franetich, J. F., Prenant, M., Belnoue, E., Rénia, L., Hannoun, L., Elings, W., Levy, S., Boucheix, C., \& Mazier, D. (2003). 
Hepatocyte CD81 is required for Plasmodium falciparum and Plasmodium yoelii sporozoite infectivity. Nature Medicine, 9, 93-96.

13. Manzoni, G., Marinach, C., Topçu, S., Briquet, S., Grand, M., Tolle, M., Gransagne, M., Lescar, J., Andolina, C., Franetich, J. F., Zeisel, M. B., Huby, T., Rubinstein, E., Snounou, G., Mazier, D., Nosten, F., Baumert, T. F., \& Silvie, O. (2017). Plasmodium P36 determines host cell receptor usage during sporozoite invasion. Elife, 6, e25903.

14. Rodrigues, C. D., Hannus, M., Prudencio, M., Martin, C., Goncalves, L. A., Portugal, S., Epiphanio, S., Akinc, A., Hadwiger, P., Jahn-Hofmann, K., Rohl, I., van Gemert, G. J., Franetich, J. F., Luty, A. J., Sauerwein, R., Mazier, D., Koteliansky, V., Vornlocher, H. P., Echeverri, C. J., \& Mota, M. M. (2008). Host scavenger receptor SR-BI plays a dual role in the establishment of malaria parasite liver infection. Cell Host and Microbe, 4, 271-282.

15. Langlois, A. C., Manzoni, G., Vincensini, L., Coppée, R., Marinach, C., Guérin, M., Huby, T., Carrière, V., Cosset, F. L., Dreux, M., Rubinstein, E., \& Silvie, O. (2020). Molecular determinants of SR-B1-dependent Plasmodium sporozoite entry into hepatocytes. Scientific Reports, 10, 13509.

16. Hall, N., Karras, M., Raine, J. D., Carlton, J. M., Kooij, T. W. A., Berriman, M., Florens, L., Janssen, C. S., Pain, A., Christophides, G. K., James, K., Rutherford, K., Harris, B., Harris, D., Churcher, C., Quail, M. A., Ormond, D., Doggett, J., Trueman, H. E., ... Sinden, R. E. (2005). A comprehensive survey of the Plasmodium life cycle by genomic, transcriptomic, and proteomic analyses. Science, 307, 82-86.

17. Roos, D. S., Kissinger, J. C., Milgram, A. J., Fraunholz, M. J., Roos, D. S., Brunk, B. P., Crabtree, J., Diskin, S. J., Schug, J., Overton, G. C., Stoeckert, C. J., Coppel, R. L., \& Huestis, R. L. (2001). PlasmoDB: An integrative database of the Plasmodium falciparum genome. Tools for accessing and analyzing finished and unfinished sequence data. Nucleic Acids Research, 29, 66-69.

18. Meier, F., Brunner, A. D., Koch, S., Koch, H., Lubeck, M., Krause, M., Goedecke, N., Decker, J., Kosinski, T., Park, M. A., Bache, N., Hoerning, O., Cox, J., Räther, O., \& Mann, M. (2018). Online parallel accumulation-serial fragmentation (PASEF) with a novel trapped ion mobility mass spectrometer. Molecular and Cellular Proteomics, 17, 2534-2545.

19. Fernández-Costa, C., Martínez-Bartolomé, S., McClatchy, D. B., Saviola, A. J., Yu, N. K., \& Yates, J. R. (2020). Impact of the identification strategy on the reproducibility of the DDA and DIA results. Journal of Proteome Research, 19, 3153-3161.

20. Tabb, D. L., Vega-Montoto, L., Rudnick, P. A., Variyath, A. M., Ham, A. J. L., Bunk, D. M., Kilpatrick, L. E., Billheimer, D. D., Blackman, R. K., Cardasis, H. L., Carr, S. A., Clauser, K. R., Jaffe, J. D., Kowalski, K. A., Neubert, T. A., Regnier, F. E., Schilling, B., Tegeler, T. J., Wang, M., ... Spiegelman, C. (2010). Repeatability and reproducibility in proteomic identifications by liquid chromatography-tandem mass spectrometry. Journal of Proteome Research, 9, 761-776.

21. Sidik, S. M., Huet, D., Ganesan, S. M., Huynh, M. H., Wang, T., Nasamu, A. S., Thiru, P., Saeij, J. P. J., Carruthers, V. B., Niles, J. C., \& Lourido, S. (2016). A genome-wide CRISPR screen in Toxoplasma identifies essential apicomplexan genes. Cell, 166, 1423-1435.e12.

22. Bargieri, D. Y., Thiberge, S., Tay, C. L., Carey, A. F., Rantz, A., Hischen, F., Lorthiois, A., Straschil, U., Singh, P., Singh, S., Triglia, T., Tsuboi, T., Cowman, A., Chitnis, C., Alano, P., Baum, J., Pradel, G., Lavazec, C., \& Ménard, R. (2016). Plasmodium Merozoite TRAP family protein is essential for vacuole membrane disruption and gamete egress from erythrocytes. Cell Host and Microbe, 20, 618-630.

23. Liffner, B., Frölich, S., Heinemann, G. K., Liu, B., Ralph, S. A., Dixon, M. W. A., Gilberger, T. W., \& Wilson, D. W. (2020). PfCERLI1 is a conserved rhoptry associated protein essential for Plasmodium falciparum merozoite invasion of erythrocytes. Nature Communications, 11, 1-14.

24. Suarez, C., Lentini, G., Ramaswamy, R., Maynadier, M., Aquilini, E., Berry-Sterkers, L., Cipriano, M., Chen, A. L., Bradley, P., Striepen, B., Boulanger, M. J., \& Lebrun, M. (2019). A lipid-binding protein mediates rhoptry discharge and invasion in Plasmodium falciparum and Toxoplasma gondii parasites. Nature Communications, 10, 1-14.

25. Uzureau, P., Barale, J. C., Janse, C. J., Waters, A. P., \& Braun Breton, C. (2004). Gene targeting demonstrates that the Plasmodium berghei subtilisin PbSUB2 is essential for red cell invasion and reveals spontaneous genetic recombination events. Cellular Microbiology, 6, 65-78.

26. Thomas, J. A., Tan, M. S. Y., Bisson, C., Borg, A., Umrekar, T. R., Hackett, F., Hale, V. L., Vizcay-Barrena, G., Fleck, R. A., Snijders, A. P., Saibil, H. R., \& Blackman, M. J. (2018). A protease cascade regulates release of the human malaria parasite Plasmodium falciparum from host red blood cells. Nature Microbiology, 3, 447-455.

27. Silvie, O., Goetz, K., \& Matuschewski, K. (2008). A sporozoite asparagine-rich protein controls initiation of Plasmodium liver stage development. PLoS Pathogens, 4, e1000086.

28. Aly, A. S. I., Mikolajczak, S. A., Rivera, H. S., Camargo, N., Jacobs-Lorena, V., Labaied, M., Coppens, I., \& Kappe, S. H. I. (2008). Targeted deletion of SAP1 abolishes the expression of infectivity factors necessary for successful malaria parasite liver infection. Molecular Microbiology, 69, 152-163.

29. Manzoni, G., Briquet, S., Risco-Castillo, V., Gaultier, C., Topcu, S., Ivanescu, M. L., Franetich, J. F., Hoareau-Coudert, B., Mazier, D., Silvie, O., Topçu, S., Ivănescu, M. L., Franetich, J. F., Hoareau-Coudert, B., Mazier, D., \& Silvie, O. (2014). A rapid and robust selection procedure for generating drug-selectable marker-free recombinant malaria parasites. Scientific Reports, 4, 4760, 1-10.

30. Yoshida, N., Nussenzweig, R. S., Potocnjak, P., Nussenzweig, V., \& Aikawa, M. (1980). Hybridoma produces protective antibodies directed against the sporozoite stage of malaria parasite. Science, 207, 71-73.

31. Perez-Riverol, Y., Csordas, A., Bai, J., Bernal-Llinares, M., Hewapathirana, S., Kundu, D. J., Inuganti, A., Griss, J., Mayer, G., Eisenacher, M., Pérez, E., Uszkoreit, J., Pfeuffer, J., Sachsenberg, T., Yilmaz, Ş., Tiwary, S., Cox, J., Audain, E., Walzer, M., ... Vizcaíno, J. A. (2019). The PRIDE database and related tools and resources in 2019: Improving support for quantification data. Nucleic Acids Research, 47, D442-D450.

\section{SUPPORTING INFORMATION}

Additional supporting information may be found online https://doi.org/10.1002/pmic.202000305 in the Supporting Information section at the end of the article.

How to cite this article: Hamada, S., Pionneau, C., Parizot, C., Silvie, O., Chardonnet, S., \& Marinach, C. (2021). In-depth proteomic analysis of Plasmodium berghei sporozoites using trapped ion mobility spectrometry with parallel accumulation-serial fragmentation. Proteomics, 21, e2000305. https://doi.org/10.1002/pmic.202000305 